\title{
La etapa posterior a la erradicación de la viruela en México, 1952-1977
}

\author{
The phase after the eradication of \\ smallpox in Mexico, 1952-1977
}

\section{Nydia Aguirre-Bolaños \\ Licenciada en Historia, Facultad de Estudios Superiores Acatlán/ Universidad Nacional Autónoma de México. \\ Atizapán de Zaragoza - Estado de México - México \\ nydia.aguirre.bolagnos@gmail.com}

Recebido em 28 abr. 2017. Aprovado em 15 jan. 2018.
AGUIRRE-BOLAÑOS, Nydia. La etapa posterior a la erradicación de la viruela en México, 1952-1977. História, Ciências, Saúde - Manguinhos, Rio de Janeiro, v.25, n.3, jul.-set. 2018, p.871-877.

\section{Resumen}

Esta nota estudia la etapa posterior a la declaración de la erradicación de la viruela en México y comprende de junio de 1952 (fecha en que las autoridades sanitarias proclamaron oficialmente este acontecimiento) a 1977 (año en que el Instituto Nacional de Higiene elaboró el último lote de vacuna antivariolosa glicerinada). Expone que durante estos años, la Secretaría de Salubridad y Asistencia continuó invirtiendo recursos en trabajos dirigidos al combate a la viruela para sostener los niveles de protección antivariolosa y evitar la reintroducción de la enfermedad en una época en que ésta todavía era endémica en varios países del mundo; y refiere el hecho de que la población siguió denunciando casos sospechosos $\mathrm{y}$ atribuyendo muertes a esta enfermedad.

Palabras clave: post-erradicación; viruela; vacunación; México.

\section{Abstract}

This paper studies the phase after the declaration of the eradication of smallpox in Mexico and covers the period from June 1952 (the date on which the health authorities officially proclaimed this event) to 1977 (the year in which the National Institute of Hygiene prepared the last batch of glycerinated antismallpox vaccine). It is revealed that during these years, the Ministry of Health and Assistance continued to invest resources in work aimed at combating smallpox to maintain the levels of protection against smallpox and to prevent the reintroduction of the disease at a time when it was still endemic in several countries worldwide; and states that the population continued to report suspected cases and attribute deaths to this disease.

Keywords: post-eradication; smallpox; vaccination; Mexico.

http://dx.doi.org/10.1590/S0104-59702018000400015 
$\mathrm{E}^{\mathrm{n}}$ n la primera mitad de 1951 ocurrieron en México la última epidemia y el último caso de viruela. Un año más tarde, el 16 de junio de 1952, la Secretaría de Salubridad y Asistencia (SSA) proclamó la erradicación de la viruela, es decir, el fin de la presencia endémico-epidémica de una enfermedad que había sido motivo de preocupación y alarma para familias, médicos y autoridades novohispanas y mexicanas durante poco más de cuatro siglos.

Aunque México logró eliminar la viruela de sus estadísticas oficiales de morbilidad y mortalidad - con poco más de $60 \%$ de la población protegida (Calderón, Celis, Pintado, 1952, p.133) -, la enfermedad seguiría ocupando un espacio tanto en el imaginario de la población como en los programas de la SSA, que siguió invirtiendo recursos en trabajos de planeación, producción de la vacuna, vacunación, vigilancia epidemiológica e investigación durante 25 años más, debido al riesgo que representaba la posible importación de casos de países que aún padecían la enfermedad. En 1959 y 1967, este padecimiento todavía era endémico en 59 y en 31 países de África, América y Asia, respectivamente en los años señalados (Fenner, 1988, p.393-394, 518); y los más relevantes para México fueron Argentina, Bolivia, Brasil, Colombia, Ecuador y Paraguay, en 1959, y Brasil, en 1967.

La historiografía sobre la lucha contra la viruela en México es amplia; sin embargo, los trabajos que se han hecho sobre los últimos años de la lucha antivariolosa y la erradicación de la viruela en este país son pocos (Bustamante, 1977; Fernández de Castro, 1997; Aguirre Bolaños, 2015; Agostoni, 2016). Hasta el momento, la historia de los años posteriores a este acontecimiento no ha sido escrita, debido a que, para muchos, la lucha contra esta enfermedad termina en 1952, con la declaración oficial de su erradicación.

La presente nota es sobre el primer trabajo que estudia lo que sucedió en México con el combate a la viruela después de haber alcanzado la erradicación de la enfermedad; ${ }^{1}$ ésta forma parte de una investigación en curso que se desprende de mi tesis de licenciatura y está basada en fuentes documentales oficiales y de prensa, que más que llegar a conclusiones plantea preguntas; expone que si bien la campaña antivariólica alcanzó el objetivo de acabar con la viruela en México, los trabajos contra esta dolencia continuaron bajo un nuevo enfoque que buscaba sostener los niveles de protección y evitar la reintroducción del padecimiento en una época en que la erradicación mundial no había sido alcanzada.

En 1949, la Oficina Sanitaria Panamericana (OSP) fue el primer organismo sanitario internacional que propuso unir fuerzas para alcanzar el objetivo de la erradicación de la viruela a nivel continental. No pasó mucho tiempo para que la Organización Mundial de Salud (OMS) comenzara a considerar las posibilidades de dicha propuesta como aspiración mundial; durante prácticamente toda la década de los años 1950 la idea de la erradicación global de la viruela ocupó un espacio en las mesas de discusión de la Asamblea Mundial de la Salud. En 1959, el Programa Global para la Erradicación de la Viruela fue aprobado y puesto en marcha, y, en 1967, asumió la modalidad de programa intensivo y de principal interés para la OMS, por lo que la Organización le asignó un presupuesto especial, y diseñó una estrategia específica para alcanzar este objetivo en un tiempo límite de diez años (Fenner, 1988, p.366-367). En 1971, Brasil registró los últimos casos de viruela en el continente americano; dos años después, en 1973, América declaró la erradicación de esta 
enfermedad. ${ }^{2}$ La erradicación de la viruela del mundo fue certificada por una comisión especial en mayo de 1980.

Luego de haber proclamado la erradicación de la viruela, la SSA propuso sostener y elevar el índice de inmunidad del país, y continuar los trabajos de vacunación de la siguiente manera: intensiva en los 15 estados que en el pasado habían presentado mayor problema varioloso, ${ }^{3}$ selectiva en los estados restantes, y rutinaria en las oficinas sanitarias del país (Programa..., 1954; Ponce de León, 1955). La atención de los programas de vacunación y revacunación antivariolosa recayó principalmente en los recién nacidos, los niños en el primer y último grado de primaria, los jóvenes entre 18 y 20 años o en Servicio Militar Nacional, el cuerpo médico y paramédico, el personal que laboraba en puertos y aeropuertos con tráfico internacional, y los empleados de hoteles (Guzmán Neyra, 1961). Asimismo, los programas reforzaron la vacunación en las zonas vecinas a los puertos fronterizos, marítimos y aéreos, y exigieron a todos los viajeros procedentes de países, infectados o no, un certificado de vacunación antivariolosa. ${ }^{4}$

En 1968 y 1970, México fue sede, respectivamente, de los Juegos Olímpicos y de la Copa Mundial de Fútbol; debido a que el país iba a recibir una gran afluencia de viajeros y turistas procedentes de países donde la viruela seguía siendo un problema, las autoridades sanitarias decidieron intensificar las actividades de vigilancia epidemiológica y llevar a cabo una campaña especial de vacunación y revacunación para proteger a la población, en particular al personal que laboraba en los aeropuertos, los hoteles contratados por la Fifa, las casas de huéspedes, los domicilios que alojarían a los extranjeros y los centros de salud.

Entre 1952 y 1961 el número total de inoculaciones antivariolosas registradas muestra un descenso considerable respecto a los años anteriores (Aguirre Bolaños, 2015, p.24). Debido posiblemente a la confianza que despertó la erradicación de la viruela, el porcentaje de padres de familia que llevaba a sus hijos a vacunar disminuyó, así como la intensidad con que esta actividad se llevaba a cabo. No obstante, la permanencia del padecimiento en otros países y las noticias sobre la introducción de casos importados que habían originado brotes de importancia entre 1961 y 1973 en algunos países de Europa, despertó después alarma ante la posibilidad de que esto pudiera ocurrir en México (Informe..., 1962) y fue un estímulo para que la población acudiera a los centros de salud para ser vacunada, o bien, para que la SSA duplicara el número de inoculaciones antivariolosas en esos años.

Aunque desde junio de 1951 la SSA no volvió a confirmar un solo caso de viruela en México, entre la población hubo rumores de casos aislados o brotes de viruela y éstos siguieron siendo noticia en periódicos. Los registros de defunciones por enfermedades infecciosas de la Dirección General de Bioestadística de la SSA reportaron muertes por viruela desde 1951 y hasta 1974 (Informe..., 1970; Informe..., 1974; Aguirre Bolaños, 2015, p.137146); pero, todas fueron negadas categóricamente por las máximas autoridades sanitarias, quienes argüían que en ningún caso las investigaciones epidemiológicas realizadas habían comprobado la existencia de la enfermedad, y que el origen de esas muertes debía atribuirse a otros padecimientos eruptivos de desenlace fatal como el sarampión o la varicela, o bien, que los certificados de dichas muertes habían sido expedidos en localidades rurales que no contaban con médicos diplomados, donde era "el encargado del Registro Civil, quien fiado en su criterio, o [en el del] familiar, [asentaba] la causa de la muerte" (Número..., 1956). No 
obstante, la población no siempre quedaba convencida con las respuestas y explicaciones que daba la SSA ante las denuncias y rumores.

México tenía a su favor que en Norteamérica, Centroamérica y el Caribe la viruela había dejado de ser endémica desde principios de la década de los 1950; mas no hay que perder de vista que la importación de casos era un peligro latente puesto que el creciente turismo y la popularización del transporte aéreo, que acortó aún más las distancias y permitió los viajes intercontinentales, facilitó también la comunicación de enfermedades como la viruela de áreas donde ésta sí era endémica a otras donde ya había sido erradicada, puesto que el tiempo de viaje era más corto que el tiempo de incubación de la enfermedad. En 1956, la viruela fue introducida por viajeros a 18 países (Coriell, 1966, p.493). Perú, que había declarado erradicada la viruela de su territorio en 1955, reportó, en 1963, la presencia de casos importados de Brasil, que dieron origen a casos secundarios. Este país pudo volver a declarar la erradicación de dicho padecimiento en 1966 (Fenner, 1988, p.595). Entre 1961 y 1973 hubo 27 casos importados en Europa, que originaron un total de 568 casos secundarios (La erradicación..., 1979, p.95).

La suspensión de la vacunación sistemática contra la viruela en México fue motivo de controversia; en 1970, 18 años después de la declaración oficial de la erradicación de la viruela, algunos médicos como Jorge Vilchis Villaseñor, opinaban que México debía continuar la vacunación antivariolosa al menos durante tres años más, y después cada año evaluar su conveniencia, ya que consideraban que la suspensión de ésta actividad implicaría la dependencia total de la seguridad antivariólica nacional en la vigilancia epidemiológica y la detección temprana de los casos, lo cual consideraban inconveniente porque "alrededor de $75 \%$ de la población, nunca ha visto un caso de viruela o lo vio cuando era muy pequeño, [y] una buena proporción de los actuales profesores de medicina y de enfermería y las últimas 17 generaciones de médicos, tampoco han visto casos" (Vilchis Villaseñor, 1971, p.151-152). Pero, en 1972, el médico Carlos Campillo Sáinz, subsecretario de Asistencia, declaró que la SSA dejaría de vacunar de manera sistemática contra la viruela debido a que hacía 20 años que en México no se presentaba un solo caso de ésta (Ya no..., 29 sept. 1972). No obstante estas declaraciones, una búsqueda exhaustiva sobre las últimas vacunaciones contra la viruela en el país indica que a pesar de que las autoridades sanitarias dijeron que ésta se suspendería en 1972, tres años después, es decir, en 1975, todavía se vacunaba en el estado de Aguascalientes; ${ }^{5}$ además, el Estado mexicano siguió produciendo vacuna antivariolosa hasta 1977 (INH, 1989), ${ }^{6}$ y destruyó sus reservas de la misma en 1982 - siguiendo las recomendaciones del doctor Isao Arita, jefe de la Unidad de Erradicación de la Viruela de la OMS (Carrillo, 2017).

En los años posteriores a la declaración oficial de la erradicación de la viruela en México, el Instituto Nacional de Higiene (INH) siguió trabajando para mejorar la calidad y el prendimiento ${ }^{7}$ de la vacuna antivariolosa glicerinada mexicana..${ }^{8}{ }^{9}$ México colaboró con el Programa Global de Erradicación de la Viruela a través del Plan Regional de Erradicación de la Viruela para el Continente Americano, coordinado por la OSP; en 1957, donó trecientas mil dosis de linfa antivariolosa para el combate contra la viruela en Haití, y entre 1959 y 1966 donó al fondo establecido por la OMS para erradicar la viruela del mundo un total de 2,400,000 dosis de vacuna, repartidas entre Brasil, Costa 
Rica, El Salvador, Honduras, Nicaragua, Panamá y República Dominicana (SSA, 1967; Fenner, 1988, p.400-401).

La erradicación de una enfermedad es una empresa ambiciosa. Aunque a mediados del siglo XX, se hablaba de la erradicación de alguna enfermedad cuando se lograba reducir a cero la incidencia del padecimiento en un área geográfica definida (Andrews, Langmuir, 1963), como sucedió en el caso que aborda esta nota; hoy en día - en parte, gracias a la experiencia de la erradicación de la viruela - la OMS distingue entre eliminación y erradicación de enfermedades. Si bien ambos términos coinciden en su objetivo - acabar para siempre con la enfermedad acordada -, difieren en el espacio geográfico que abarcan; es decir, mientras, la primera se puede aplicar a un país, una región o un continente, la segunda extiende su alcance a todo el mundo, y, por tanto, adquiere un carácter absoluto y permanente, y en ella intervienen dos factores fundamentales: la supresión del agente patógeno y la suspensión de cualquier medida sanitaria contra la enfermedad erradicada (Dowdle, 1998; Barrett, 2004).

En México, la erradicación de la viruela se celebró como un logro de la salud pública nacional, con fuertes implicaciones políticas y patrióticas; para algunos médicos ésta constituía "la resolución a fondo de un problema nacional" (Bustamante, 1977, p.573), y se enorgullecían de haber logrado ese objetivo con vacuna glicerinada producida por el Instituto de Higiene y aplicada por vacunadores mexicanos mediante las técnicas tradicionales de multipresión o escarificación y, en la última etapa, sin la ayuda de la OSP. ${ }^{10}$

La supresión de la enfermedad y su renovada ausencia introdujeron cambios en la lucha y en la percepción sobre esta enfermedad. No obstante, el elevado número de reportes despierta sospechas en cuanto a si realmente no hubo un solo caso nativo o importado de viruela en México después de la proclamación de su erradicación; ya que si realmente el último caso de viruela en México hubiera ocurrido en junio de 1951, y todos los casos posteriores reportados no hubieran sido ciertos, dichos reportes manifestarían la permanencia de la viruela en el imaginario de aquellas generaciones que crecieron y convivieron con ella, para quienes esta enfermedad no habría desaparecido por decreto de un día para otro; en caso contrario, si alguno de esos casos sí lo hubiera sido, la negativa de las autoridades mexicanas para hacerlo público manifestaría el peso que se le atribuía al logro de la erradicación de la viruela, ya que las autoridades quizá pensaban que admitir la existencia de un solo caso de viruela podría debilitar la credibilidad, el prestigio y la autoridad de la SSA y el Estado mexicano ante la población y el concierto de naciones. Sin embargo, este es un asunto que todavía debo corroborar, y uno de los medios que he pensado para hacerlo es a través de las fuentes orales - entrevistas a médicos y a posibles atacados por esta enfermedad.

\section{NOTAS}

${ }^{1}$ La presente nota de pesquisa forma parte de una investigación más amplia, mi tesis de licenciatura: $L a$ alineación de los planetas: erradicación de la viruela en México, 1941-1977, disponible para su consulta en: <http://132.248.9.195/ptd2015/noviembre/303785099/303785099.pdfsearch=\%22viruela\%22>.

${ }^{2}$ En Norteamérica la viruela dejó de ser un problema a principios de la década de los 1950; en Centroamérica y algunas islas del Caribe la presencia de la misma era esporádica, no endémica. Los países de América del 
Sur alcanzaron la erradicación de la viruela durante las décadas de los 1950 y 1960: Chile en 1954, Perú en 1955, Venezuela en 1956, Uruguay en 1957, Bolivia y Paraguay en 1960, Ecuador en 1963, Colombia en 1965, Argentina en 1966 y Brasil en 1971 (Fenner, 1988, p.327, 329, 333).

${ }^{3}$ Que comprendía al Distrito Federal y a los estados de Aguascalientes, Guanajuato, Guerrero, Hidalgo, Jalisco, Estado de México, Michoacán, Oaxaca, Puebla, Querétaro, San Luis Potosí, Tlaxcala, Veracruz y Zacatecas (Calderón, Celis, Pintado, 1952, p.132).

${ }^{4}$ El Certificado Internacional de Vacunación o Revacunación contra la Viruela fue aprobado por la cuarta Asamblea Mundial de la Salud en mayo de 1951. No obstante, México y EEUU habían acordado dicho requisito desde 1947.

${ }^{5}$ En 1975, el gobernador del estado de Aguascalientes reportó que ese año se habían practicado 5.658 vacunaciones y 7.471 revacunaciones (Primer..., 1975).

${ }^{6}$ En 1955, el INH producía más de 12 millones de dosis de vacuna antivariolosa (Ponce de León, 1955, p.89); en 1961 dicha producción se redujo a ocho millones (Conclusiones..., 1961), y en 1975 ésta fue de tan solo dos millones (SSA, 1977).

${ }^{7}$ Palabra que se usaba para referir el éxito y la actividad de la vacuna, el cual se reconocía por la evolución y marca que ésta dejaba en el brazo del vacunado luego de ser aplicada.

${ }^{8}$ A principios de la década de los 1950, el porcentaje de prendimiento de la vacuna era de $90 \%$ (Comunicación..., 1950); pero, en 1963, la misma, llegó a alcanzar entre 94 y 100\% (Informe..., 1962).

${ }^{9}$ Aunque el INH realizó algunas pruebas con vacuna desecada, éstas no lograron competir con la antigüedad de la vacuna glicerinada y con la pericia que tenían los técnicos mexicanos en la producción de la misma, la cual siempre fue "el biológico de elección por su fácil manejo, su conocimiento nacional y su bajo costo" (Informe..., 1962).

${ }^{10}$ En 1950, un año después de que la OSP aprobara la propuesta de su director para unir fuerzas contra la viruela en América, la SSA sostuvo comunicaciones con ella para desarrollar un convenio de cooperación; no obstante, México rechazó el acuerdo propuesto por considerarlo poco benéfico para la lucha antivariolosa (Aguirre Bolaños, 2015, p.54-61).

\section{REFERENCIAS}

AGOSTONI, Claudia.

Médicos, campañas y vacunas: la viruela y la cultura de su prevención en México 1870-1952. México: Unam/Instituto de Investigaciones Dr. José María Luis Mora. 2016.

AGUIRRE BOLAÑOS, Nydia.

La alineación de los planetas: erradicación de la viruela en México, 1941-1977. Tesis (Licenciatura en Historia) - Universidad Nacional Autónoma de México, Ciudad de México. 2015.

ANDREWS, Justin M.; LANGMUIR, Alexander D. The philosophy of disease eradication. American Journal of Public Health, v.53, n.1, p.1-6. 1963.

BARRETT, Scott.

Eradication versus control: the economics of global infectious disease policies. Bulletin of the World Health Organization, v.82, n.9, p.683-688. 2004.

BUSTAMANTE, Miguel E.

Consecuencias médico-sociales de la viruela y de su erradicación. Gaceta Médica de México, v.113, n.12, p.564-573. 1977.
CALDERÓN, Carlos; CELIS, Heliodoro; PINTADO, Ramón.

Supresión del estado endemo-epidémico de la viruela en México. Boletín de la Oficina Sanitaria Panamericana, v.33, n.2, p.128-134. 1952.

CARRILLO, Ana María.

Vaccine production, national security anxieties and the unstable State in nineteenth and twentieth century Mexico. In: Holmberg, Christine; Blume, Stuart; Greenough, Paul. The politics of vaccination: a global history. Manchester: Manchester University Press. p.121147. 2017.

\section{COMUNICACIÓN...}

Comunicación entre la SSA y la OSP. Secretaría de Salubridad y Asistencia. Subsecretaría de Asistencia; c.31, e.3, 257fs (Archivo Histórico de la Secretaría de Salud, Ciudad de México). 1950.

CONCLUSIONES...

Conclusiones de la Junta del Comité Antivarioloso de 1961. Secretaría de Salubridad y Asistencia. Secretaría Particular; c.79, e.2, s.f. (Archivo Histórico de la Secretaría de Salud, Ciudad de México). 1961. 
CORIELL, Lewis $\mathrm{L}$.

Smallpox vaccination: when and whom to vaccinated. Pediatrics, v.37, n.3, p.493-496. 1966.

DOWDLE, Walter R.

The principles of disease elimination and eradication. Bulletin of the World Health Organization, v.76, s.2, p.22-25. 1998.

FENNER, Frank et al. Smallpox and its eradication. Ginebra: WHO. 1988.

FERNÁNDEZ DE CASTRO, Jorge.

La erradicación de la viruela en México. Gaceta Médica de México, v.133, n.2, p.99-106. 1997.

GUZMÁN NEYRA, Edmundo. Instructivo para la vacunación antivariólica permanente. Boletín Epidemiológico, v.25, n.4, p.132. 1961.

INFORME...

Informe de actividades desarrolladas por la Dirección de Epidemiología y Campañas Sanitarias. Instituto Nacional de Higiene. Trabajos de concurso; c.2, e.22, f.445 (Archivo Histórico de la Secretaría de Salud, Ciudad de México). 1974.

INFORME...

Informe de actividades desarrolladas por la Dirección de Epidemiología y Campañas Sanitarias. Secretaría de Salubridad y Asistencia. Subsecretaría de Salubridad; c.8, e.2, s.f. (Archivo Histórico de la Secretaría de Salud, Ciudad de México). 1970.

INFORME...

Informe de actividades desarrolladas por la Dirección de Epidemiología y Campañas Sanitarias. Secretaría de Salubridad y Asistencia. Subsecretaría de Asistencia; c.92, e.1, s.f. (Archivo Histórico de la Secretaría de Salud, Ciudad de México). 1962.

INH.

Instituto Nacional de Higiene. Trabajos de concurso; c.2, e.22, s.f. (Archivo Histórico de la Secretaría de Salud, Ciudad de México). 1989.
LA ERRADICACIÓN...

La erradicación mundial de la viruela: informe final de la Comisión Mundial para la Certificación de la Erradicación de la Viruela. Ginebra: OMS. 1979.

NÚMERO...

Número de defunciones causadas por ciertas enfermedades transmisibles. Secretaría de Salubridad y Asistencia. Subsecretaría de Salubridad y Asistencia; c.31, e.2, 823fs (Archivo Histórico de la Secretaría de Salud, Ciudad de México). 1956.

PONCE DE LEÓN, Alfonso.

Viruela: su erradicación. Boletín Epidemiológico, v.19, n.3, p.88-89. 1955.

PRIMER...

Primer informe de gobierno que rinde el gobernador constitucional del estado de Aguascalientes. Periódico Oficial del Estado de Aguascalientes, v.38, n.48, p.13. 1975.

PROGRAMA...

Programa de labores de la Dirección de Epidemiología. Secretaría de Salubridad y Asistencia. Subsecretaría de Salubridad y Asistencia; c.24, e.3, s.f. (Archivo Histórico de la Secretaría de Salud, Ciudad de México). 1954.

SSA.

Secretaría de Salubridad y Asistencia. Gerencia General de Biológicos y Reactivos; c.17, e.6, 219fs (Archivo Histórico de la Secretaría de Salud, Ciudad de México). 1977.

SSA.

Secretaría de Salubridad y Asistencia. Oficina de Asuntos Internacionales; c.52, e.6, s.f. (Archivo General de la Nación, Ciudad de México). 1967.

VILCHIS VILLASEÑOR, Jorge.

Prevención de las enfermedades transmisibles en México: viruela. Gaceta Médica de México, v.101, n.2, p.144-153. 1971.

YA NO...

Ya no se vacunarán contra la viruela por sistema. El Informador, p.1-A. 29 sept. 1972.

\section{$\rightarrow \rightarrow \rightarrow<<<$}

